P. Nau, P. Kutne, G. Eckel, W. Meier, C. Hotz, S. Fleck, Infrared absorption spectrometer for the determination of temperature and species profiles in an entrained flow gasifier, Appl Opt 56 (2017) 2982-2990.

This paper was published in Applied Optics and is made available as an electronic reprint with the permission of OSA. The paper can be found at the following URL on the OSA website: [https://doi.org/10.1364/AO.56.002982].

(C) 2017 Optical Society of America, Inc. 


\title{
Infrared absorption spectrometer for the determination of temperature and species profiles in an entrained flow gasifier
}

\author{
Patrick Nau ${ }^{1}$, Peter Kutne ${ }^{1}$, Georg Eckel ${ }^{1}$, Wolfgang Meier ${ }^{1}$, Christian Hotz $^{2}$, And \\ SABINE FLECK ${ }^{2}$ \\ ${ }^{1}$ German Aerospace Center (DLR), Institute of Combustion Technology, Pfaffenwaldring 38-40, 70569 Stuttgart, Germany \\ ${ }^{2}$ Karlsruhe Institute of Technology (KIT), ITC, Hermann-von-Helmholtz-Platz 1, 76344 Eggenstein-Leopoldshafen, Germany \\ ${ }^{*}$ Corresponding author: patrick.nau@dlr.de
}

Compiled February 10, 2017

\begin{abstract}
An absorption spectrometer utilizing a tunable distributed feedback diode laser at $2.3 \mu \mathrm{m}$ and an interband cascade laser at $3.1 \mu \mathrm{m}$ has been developed to measure temperature and concentrations of $\mathrm{CO}$, $\mathrm{CH}_{4}, \mathrm{C}_{2} \mathrm{H}_{2}$ and $\mathrm{H}_{2} \mathrm{O}$ under gasification conditions. A wavelength division multiplexing approach using a single $\mathrm{ZrF}_{4}$-fiber was used to measure both wavelength regions simultaneously. The performance of the spectrometer has been tested in laminar flat flames and a heated cell and then applied for measurements at an atmospheric entrained flow gasifier (REGA). A water cooled optical probe was used to provide optical access at two measurement positions. By moving the burner, axial profiles of temperature and species concentration could be obtained. These profiles were compared with numerical simulations and can be used to validate the simulation. (๑) 2017 Optical Society of America
\end{abstract}

OCIS codes: (300.1030) Absorption; (300.6260) Spectroscopy, diode lasers;(300.6340) Spectroscopy, infrared.

http://dx.doi.org/10.1364/ao.XX.XXXXXX

\section{INTRODUCTION}

Entrained flow gasification is a process to produce synthesis gas from a fuel, like biomass or coal, at high temperatures. The synthesis gas can then be used to produce liquid fuels or other chemicals. Gasification is a rather complex process. Therefore numerical simulations and experimental investigations are necessary to understand and improve the gasification process.

For the characterization of gasification processes and for comparison with numerical simulations, accurate measurements of temperature and species concentrations are necessary. Important species in gasification processes include $\mathrm{CO}, \mathrm{H}_{2} \mathrm{O}, \mathrm{H}_{2}$ and $\mathrm{CO}_{2}$ as the main products. In addition hydrocarbons like methane can be found. These molecules are usually intermediates and only a minor product of the gasification process. Optical measurements are the preferred technique to measure these quantities in situ, as they are nonintrusive. The limited optical access and the harsh conditions in gasification reactors make infrared absorption spectroscopy in particular very useful for this application. Successful measurements with this technique have for example been demonstrated in the particle laden atmosphere of a coal-fired power plant[1] and a fluidized bed combustor[2]. Ortwein et. al. used a vertical cavity surface-emitting laser (VCSEL) for the in situ detection of $\mathrm{HCl}$ in an atmospheric gasifier[3]. Extensive work has been done by the group of Hanson. The synthesis gas composition in the exit section of a high pressure coal gasifier $[4,5]$ and also temperature and species concentration in the reactor chamber at pressures up to $18 \mathrm{~atm}$ [6] have been measured. The high pressures in the reactor make measurements challenging, because of the pressure broadening of the absorption lines. To permit quantitative measurements the pressure broadening coefficients have therefore been measured in laboratory experiments. Sepman et. al. recently developed a sensor based on a single diode laser at $2.3 \mu \mathrm{m}$ to measure soot volume fraction, $\mathrm{CO}$ and water in an atmospheric gasifier fired with peat powder.[7] Also the influence of fuel composition and burner configuration on the soot volume fraction was investigated.[8] Extinction measurements were performed with two laser diodes at $808 \mathrm{~nm}$ and $450 \mathrm{~nm}$.

Due to the limited optical access most publications report only on measurements at one or two fixed measurement locations in the gasifier. These publications focus mainly on online process control, but for validation of numerical simulations spatially resolved profiles were desirable. Therefore the goal of this study was to measure axial profiles of the gas temperature, as well as the concentration of $\mathrm{CO}$, water 
and small hydrocarbons in the gasifier. The obtained data can then be used for validation of numerical simulations, which can then be used to improve the gasification process.

One of the main components in the produced synthesis gas is $\mathrm{CO}$. We selected the first overtone band of $\mathrm{CO}$ around $2.3 \mu \mathrm{m}$ for the measurements. This wavelength is accessible with distributed feedback (DFB) tunable diode lasers. A thorough survey of the absorption lines of $\mathrm{CO}$ has been performed to identify the most promising candidates. The goal of this survey was to identify lines that have minimal interference with other absorbing molecules like water and $\mathrm{CO}_{2}$ and the selected lines should also be used to infer the temperature. Therefore a line pair within the scanning range of the DFB laser (about $2 \mathrm{~cm}^{-1}$ ) was desirable.

In addition to $\mathrm{CO}$ a suitable wavelength for the detection of small hydrocarbons (i.e. methane and acetylene) was identified. A promising spectral window at $3.1 \mu \mathrm{m}$ was selected. In addition lines of water, which occurs in high quantities in the gasifier, can be found in this wavelength region. The selected lines provide minimized interference with other molecules to give high sensitivity and reliability. Conventional quartz windows are still useable for this wavelength region. Wavelengths around $3 \mu \mathrm{m}$ were not easily accessible with diode lasers in the past. While conventional tunable diode lasers are limited to the near infrared, quantum cascade lasers cover the wavelengths above about $4 \mu \mathrm{m}$ in the mid infrared. Interband cascade lasers (ICL) fill this gap and are therefore ideally suited for absorption spectroscopy in this wavelength region[9-11].

The selected wavelengths allow determination of mole fractions and temperature at the same time. These values can then be compared with model calculations to validate the numerical simulation. In this paper height resolved measurements in an atmospheric gasifier will be presented and compared with numerical simulations.

\section{EXPERIMENTAL AND DATA EVALUATION}

\section{A. Line selection}

To identify the most promising wavelength region for temperature and $\mathrm{CO}$ concentration measurements, simulations of the absorption spectrum of $\mathrm{CO}$ based on the HITEMP database[12] have been performed. The constraints for a suitable wavelength region are minimized interference with other molecules (mainly water, $\mathrm{CO}_{2}$ and methane), a line pair within the scanning range of a single laser diode (typically $2 \mathrm{~cm}^{-1}$ ) and sufficiently high line strengths to allow measurements with a high signal to noise ratio. The line pair R27 $\left(\mathrm{v}^{\prime \prime}=1\right)$ and R6 $\left(\mathrm{v}^{\prime \prime}=0\right)$ at $4285 \mathrm{~cm}^{-1}$ of the first overtone band turned out to be the most promising candidate (fig. 1). This line pair has already been used in the past to measure $\mathrm{CO}$ concentrations and temperatures [13]. The error in the line strength in the HITEMP database is $2-5 \%$ for both lines. These lines have lower state energies E" of $3579.97 \mathrm{~cm}^{-1}$ and $80.74 \mathrm{~cm}^{-1}$, respectively. Due to the high difference in lower state energy this line pair is well suited for temperature measurements. The intensity ratio $\mathrm{R}$ of the two lines and the resulting sensitivity factor $d R / R / d T / T$ is shown in fig. 2. In the temperature range of interest (1000$2000 \mathrm{~K})$ this line pair shows a very good sensitivity enabling precise temperature measurements. Taking into account the error in the spectroscopic constants in HITEMP and the error in determining the area of the absorption lines, the uncertainty in the measured temperature determined from the intensity ratio is estimated to $2-3 \%$. The selected line pair is free from interference with other molecules. Some weak lines of water are present in the vicinity of the $\mathrm{CO}$ lines, but do not interference with the $\mathrm{CO}$ lines (compare fig. 6a).

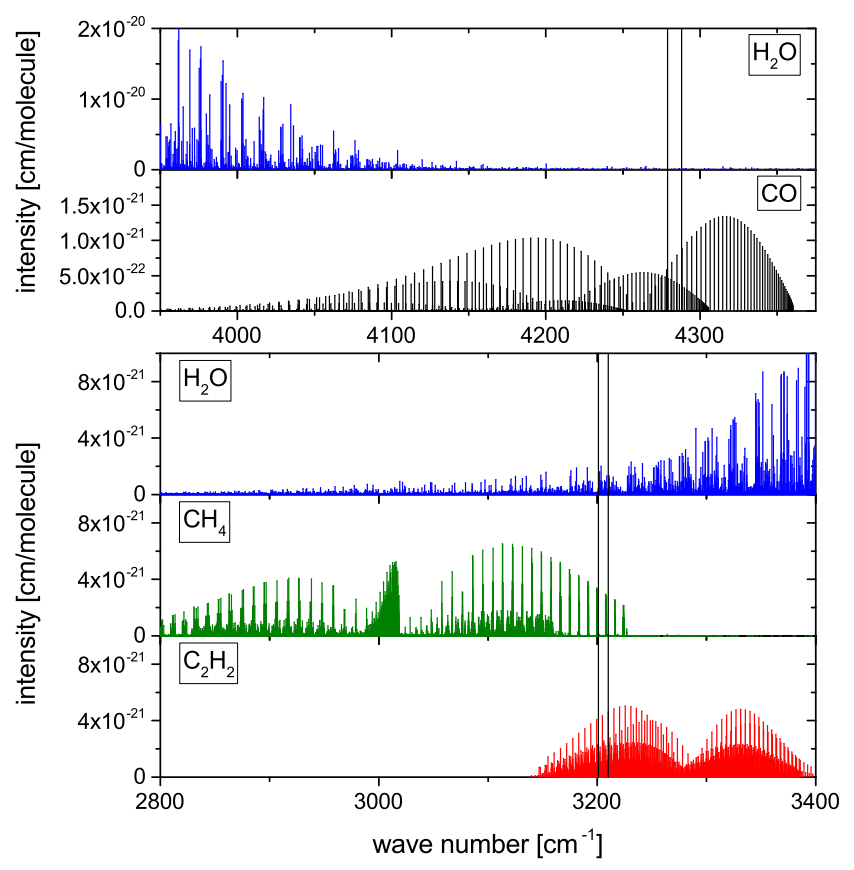

Fig. 1. Line intensities of the investigated molecules at $1500 \mathrm{~K}$ : $\mathrm{CO}$ and $\mathrm{H}_{2} \mathrm{O}$ around $2.3 \mu \mathrm{m}$ (top) and $\mathrm{C}_{2} \mathrm{H}_{2}, \mathrm{CH}_{4}$ and $\mathrm{H}_{2} \mathrm{O}$ around $3.1 \mu \mathrm{m}$ (bottom). The wavelength scanning ranges of the selected lasers have been marked with vertical bars.

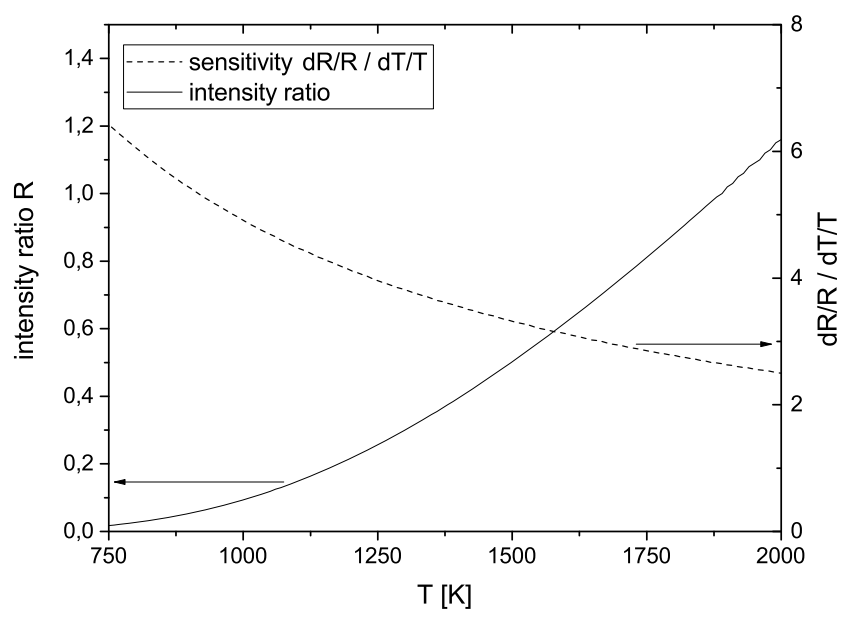

Fig. 2. Intensity ratio and temperature sensitivity of the $\mathrm{CO}$ line pair R27/R6 in the first overtone band around $4285 \mathrm{~cm}^{-1}$.

The second wavelength region was mainly chosen to measure small hydrocarbons, namely acetylene and methane. Previous investigations show methane concentration levels around $0.1 \mathrm{vol} \%$ and even lower levels for acetylene[14, 15]. These molecules have strong lines around $3 \mu \mathrm{m}$. Line intensities in the fundamental band are more than one order of magnitude stronger than in the weaker bands below $2.5 \mu \mathrm{m}$. Therefore higher sensitivities can be expected. Furthermore 
the fundamental bands of hydrocarbons around $8 \mu \mathrm{m}$ are more difficult to access, because quartz and sapphire is not transparent anymore and window materials like $\mathrm{CaF}_{2}$ are not well suited for the harsh environments in a gasifier. The absorption bands around $3 \mu \mathrm{m}$ therefore seem to be the best choice.

Simulations of the absorption spectrum based on the HITRAN and HITEMP database (line strengths and air and self pressure broadening parameters) with expected temperatures and species mole fractions $\left(1500 \mathrm{~K}, 1 \mathrm{bar}, \mathrm{H}_{2} \mathrm{O}: 30 \mathrm{vol} \%\right.$, CO: $20 \mathrm{vol} \%, \mathrm{CO}_{2}$ : $20 \mathrm{vol} \%, \mathrm{C}_{2} \mathrm{H}_{2}: 0.1 \mathrm{vol} \%, \mathrm{CH}_{4}: 0.1 \mathrm{vol} \%$ ) have been performed. The strongest interfering species in the $3 \mu \mathrm{m}$ region is water. Due to careful selection of the wavelength region, spectral windows could be found which allow the detection of acetylene, methane and water with the same laser. For acetylene a transition at $3204.7 \mathrm{~cm}^{-1}$ was identified as the most promising candidate, which is dominated by the P31e line (compare fig. 1 and fig. 6c). Based on the simulations this line gives the highest sensitivity for the expected small concentration levels of acetylene. The error in line strength in the HITRAN database[16] is $2-5 \%$ for this line. At higher wavenumbers some stronger acetylene lines are present, but also absorption from water gets stronger. Especially the Rbranch of the acetylene band shows strong interference with water and is therefore not well suited for our application. However, different applications may find other wavelengths to be better suited. Stranic and Hanson for example successfully used a line in the R-branch at $3335.55 \mathrm{~cm}^{-1}$ for pyrolysis studies in shock tubes[17].

Close by at $3208 \mathrm{~cm}^{-1}$ lines of methane are present ( 13 band, R20), which enable the possibility to measure both molecules with the same laser (compare fig. 1 and fig. 6b). The methane lines have a ground state energy E" of about $2180 \mathrm{~cm}^{-1}$. This results in low sensitivity at room temperature and highest line strength at about $740 \mathrm{~K}$, which makes these lines suitable for measurements in high temperature environments. Sur et. al. recently identified a cluster of lines near $3148.8 \mathrm{~cm}^{-1}$ as optimal for methane detection for temperatures between $900-1400 \mathrm{~K}$ in shock tube studies[9]. Although this wavelength can give higher sensitivity for methane, the lines used in this work have still sufficient sensitivity for the expected mole fractions around $0.1 \mathrm{vol} \%$. Also at lower wave numbers it would not have been possible to measure acetylene and methane with the same laser. An additional laser would have been necessary, which would further complicate the experimental setup. The HITRAN database states an error range of 10-20\% for the line strength of these lines. Water lines in this region have also high ground state energies (typically $>2000 \mathrm{~cm}^{-1}$ ) therefore minimizing absorption from water in room air, which could affect the measurement accuracy. The uncertainty of the line strength of the water lines is $5-10 \%$.

\section{B. Laser absorption spectrometer}

Two lasers were used to measure $\mathrm{CO}$ at $2.3 \mu \mathrm{m}$ and acetylene, methane and water at $3.1 \mu \mathrm{m}$. Wavelength division multiplexing was applied to measure both spectral regions simultaneously. At $2.3 \mu \mathrm{m}$ a distributed feedback (DFB) diode laser was used (nanoplus $\mathrm{GmbH}, 16 \mathrm{~mW}$ maximum output power), while at $3.1 \mu \mathrm{m}$ an ICL (nanoplus $\mathrm{GmbH}$, $5.5 \mathrm{~mW}$ maximum output power) was utilized. Both lasers are thermoelectrically stabilized and driven with a sawtooth ramp to scan the wavelength of the laser beams. In case of $\mathrm{CO}$ at $2.3 \mu \mathrm{m}$ the laser temperature was stabilized at $16^{\circ} \mathrm{C}$.
For acetylene and methane the laser temperature was set to $36^{\circ} \mathrm{C}$ and $26^{\circ} \mathrm{C}$ respectively, because both transitions could not be covered in a single scan of the laser. The sawtooth ramps at $500 \mathrm{~Hz}$ are generated with a digital I/O board (NationalInstruments, PCI6115) and used to modulate the current output of the two laser drivers (Thorlabs, LDC8002). The output of each laser is collimated with a lens and passed through an optical isolator (Thorlabs IO-4-3120-VLP and IO5-2335-VLP) to avoid optical feedback. The laser beams are overlapped with a dichroic mirror and coupled with an off-axis parabolic mirror into a $\mathrm{ZrF}_{4}$ single mode fiber (Thorlabs, P323Z-FC-2, $2 \mathrm{~m}$ long). The total efficiency of the fiber coupling is about $13 \%$. The core diameter of the fiber is $9 \mu \mathrm{m}$ and offers single mode operation from 2.3 to $4.1 \mu \mathrm{m}$. $\mathrm{A} \mathrm{CaF}_{2}$ beamsplitter is used to direct a small fraction of the overlapped laser beams through a germanium etalon (free spectral range $=0.016 \mathrm{~cm}^{-1}$ ). After the etalon the beam is focused with an off-axis parabolic mirror onto an infrared detector (VIGO Systems, PVI3TE-5). The same etalon is used for both lasers. To measure the tuning characteristics of one laser the second laser is turned off. As the laser output is very stable it is not necessary to measure an etalon trace for every tuning ramp. The laser, fiber coupling optics and etalon are housed inside a small box to provide easy transport of the system and protection of the optics. A schematic of the setup is shown in fig. 3 .

Both laser beams are guided with the $\mathrm{ZrF}_{4}$-fiber to the experiment and recollimated with an off-axis parabolic mirror to provide a laser beam with about $2 \mathrm{~mm}$ diameter. The laser beam passes through the reactor and is coupled into a second fiber (art photonics $\mathrm{GmbH}$, multimode CIR fiber with $250 \mu \mathrm{m}$ core diameter, $2 \mathrm{~m}$ long, $10 \%$ transmission) at the end of a second probe. Both beams are coupled into this fiber with an off-axis parabolic mirror and are directed to a second box which houses the signal collection optics. The signal beams are again recollimated in the detection box with a reflective collimator. A dichroic mirror is used to direct the $2.3 \mu \mathrm{m}$ beam onto an extended InGaAs detector (Thorlabs, PDA10D), while the remaining $3.1 \mu \mathrm{m}$ beam is detected with a thermoelectrically cooled IR detector (VIGO Systems, PVI-3TE-5).

\section{Data acquisition and analysis}

The etalon signal described in $2 . B$ is used to quantify the relative tuning of the laser during the modulation ramp, while the absolute line position is based on the line positions in the HITRAN database (acetylene, methane) [16] and HITEMP database ( $\mathrm{CO}$, water) [12]. To extract the number density from the measured absorption spectra an extended Lambert-Beer's law is used:

$$
\alpha_{\tilde{v}}=\ln \frac{I}{T(t) I_{0}+E(t)}=l \sum_{m} N_{m} \times \sigma_{m}(T) \times g(\tilde{v}, T, p)
$$

The measured absorption $\alpha_{\tilde{v}}$ depends on the ratio of measured laser intensity $I$ and the initial laser intensity $I_{0}$, which is affected by variation of the transmission $T(t)$ and background emission $E(t)$ like thermal radiation. Variations in the background are determined from the detector signal between individual signal ramps and subtracted from the measured signal. To account for variations in the baseline of the spectrum a third order polynomial was fitted to the signal. The absorption signal is directly proportional to the path length $l$ and depends on the sum of the absorption of the individual lines $m$ with corresponding molecular number density $N_{m}$, integrated line strength of the given line $\sigma_{m}(T)$ 


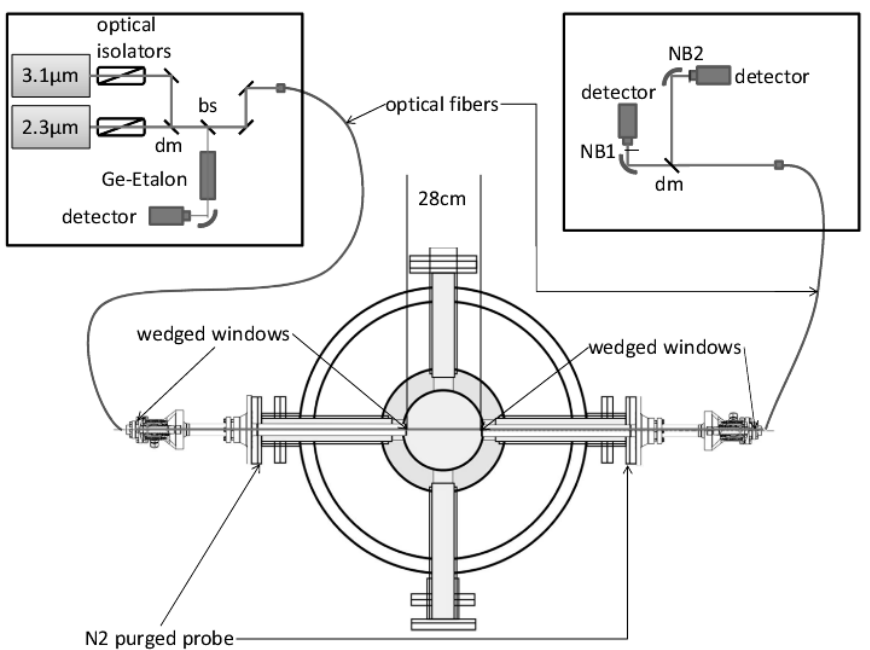

Fig. 3. Experimental setup for absorption measurements at the gasifier. Both laser beams pass an optical isolator, are overlapped with a dichroic mirror $(\mathrm{dm})$ and coupled into a single mode $\mathrm{ZrF}_{4}$ fiber. After passing the reactor the light is coupled into a second fiber and guided to the detection system. The signal beams are split with a second dichroic mirror and send to separate detectors equipped with narrow bandpass filters (NB).

and the normalized individual line shape function $g_{m}(\tilde{v}, T, p)$. Therefore the number density can be directly calculated from the integrated line strength if the line strength is known. The line shape function is modeled with a Voigt line profile[18] in this work. The Gaussian contribution to the line is calculated from the temperature and molar mass of the molecule. Pressure broadening however is more difficult to model, because it depends on the surrounding gas and the HITRAN and HITEMP databases contain only the parameters for air- and self-broadening. The Lorentzian contribution is therefore fitted for every individual line. The temperature dependence of the absorption lines depends on the lower state Energy $E^{\prime \prime}$ and the partition function $Q(T)$ of the line. Both parameters are taken from the HITRAN/HITEMP database. The temperature dependent line strength is then calculated from:

$$
\begin{array}{r}
\sigma(T)=\sigma\left(T_{0}\right) \frac{Q\left(T_{0}\right)}{Q(T)}\left(\frac{T_{0}}{T}\right) \exp \left[-\frac{h c E^{\prime \prime}}{k}\left(\frac{1}{T}-\frac{1}{T_{0}}\right)\right] \times \\
{\left[1-\exp \left(-\frac{h c \tilde{v}_{0}}{k T}\right)\right] /\left[1-\exp \left(-\frac{h c \tilde{v}_{0}}{k T_{0}}\right)\right]}
\end{array}
$$

where $h$ is Planck's constant, $c$ is the speed of light, $k$ is the Boltzmann constant and $\tilde{v}_{0}$ the center frequency of the line.

\section{Gasifier measurements}

A schematic of the experimental setup for the gasifier measurements is shown in figure 3. Measurements were performed at the atmospheric, pilot scale entrained flow gasifier REGA (Research Entrained Flow Gasifier) in Karlsruhe. The vertically arranged reactor consists of a ceramic tube with an inner diameter of $280 \mathrm{~mm}$ and a length of $3 \mathrm{~m}$. Reactor walls are heated to $1195^{\circ} \mathrm{C}$ to reduce heat loss. Flanges along the reactor axis provide access for sampling and optical measurements. The burner can be moved along the reactor axis to measure at different distances from the burner. Two reactor access locations were used to cover distances to the burner from $20-250 \mathrm{~mm}$ and $300-680 \mathrm{~mm}$ respectively. Tomographic measurements are not possible in the reactor. Therefore only line-of-sight integrated absorption measurements were carried out. The operating conditions of the reactor are shown in table 1.

Table 1. Operation conditions of the gasifier. The fuel is ethylene glycol and the oxidizer oxygen enriched air. $\lambda$ is the air-to-fuel equivalence ratio. Gas flow rates are given at $273 \mathrm{~K}$ and 1 bar.

\begin{tabular}{ccc}
\hline & Condition 1 & Condition 2 \\
\hline fuel $[\mathrm{kg} / \mathrm{h}]$ & 12.5 & 12.4 \\
Air $\left[\mathrm{m}^{3} / \mathrm{h}\right]$ & 7.0 & 2.9 \\
$\mathrm{O}_{2}\left[\mathrm{~m}^{3} / \mathrm{h}\right]$ & 5.0 & 4.6 \\
$\lambda$ & 0.57 & 0.47 \\
\hline
\end{tabular}

To ensure a defined optical path from the outside reactor flange to the inner reactor tube, two water cooled probes are used. The length of the probes is $90 \mathrm{~cm}$ and the diameter of the optics inside the probe is $25 \mathrm{~mm}$. Both probes are equipped with quartz windows at the input and output side. To keep the windows inside the reactor clean from particles and soot, the windows at the probe tip are purged with dry nitrogen $\left(0.5 \mathrm{~m}^{3} / \mathrm{h}\right)$. The nitrogen also helps to avoid absorption of the laser beams before entering the reactor chamber. A detailed description of the general probe design can be found in [19]. In contrast to that design the probe used in this investigation can be rotated and shifted back and forth freely inside the packing of the flange. Also all windows have a $2^{\circ}$ wedge to minimize etaloning, which would introduce disturbing interference signals. In addition the small angle introduced from the wedged window can be used to align the laser beam by rotating one probe relative to the second one.

To quantify the influence of the purge gas on the absorption measurements results from laser induced breakdown spectroscopy (LIBS) have been used. A Nd:YAG laser at $1064 \mathrm{~nm}(10 \mathrm{~Hz}, 200 \mathrm{~mJ})$ is used to create a plasma at a distance of $150 \mathrm{~mm}$ from the probe tip. Details of the setup can be found in [20]. The plasma signal is then used to determine the oxygen volume fraction and therefore can be used to determine the amount of nitrogen dilution. The probe can be moved in and out to move the position of the laser induced plasma inside the reactor. This way it is possible to measure at different distances from the reactor center. For measurements in the center of the reactor the probe tip is flush with the reactor wall, while at $130 \mathrm{~mm}$ from the reactor center $(10 \mathrm{~mm}$ from the reactor wall) the probe is moved out by $130 \mathrm{~mm}$.

\section{E. Validation measurements}

In addition to the measurements at the gasifier, validation measurements were performed in a set of laminar flat flames with different stoichiometries and flow conditions to cover a large range of temperatures and $\mathrm{CO}$ concentration levels. Flame conditions for the investigated flames are shown in table 2. Temperatures in these flames have been measured with Coherent anti-Stokes Raman Scattering (CARS) and the gas composition was calculated with Gaseq[21] based on the measured temperatures.[22] 
Table 2. Flame conditions for the investigated laminar flat flames. Flames were stabilized on a flat flame burner (Holthuis \& Associates) with a bronze matrix of $60 \mathrm{~mm}$ diameter. Reference temperature and $\mathrm{CO}$ mole fraction are taken from [22].

\begin{tabular}{cccccc}
\hline & $\mathrm{CH}_{4}$ & Air & & $\begin{array}{c}\text { T (CARS) } \\
{[\mathrm{K}]}\end{array}$ & $\begin{array}{c}\text { Calculated CO } \\
\text { mole fraction }\end{array}$ \\
\hline 202 & 1.31 & 12.40 & 1.0 & 1790 & 0.0008 \\
203 & 1.31 & 11.31 & 1.1 & 1754 & 0.0223 \\
220 & 1.31 & 10.40 & 1.2 & 1723 & 0.0406 \\
221 & 1.73 & 16.50 & 1.0 & 1886 & 0.0016 \\
205 & 1.73 & 15.00 & 1.1 & 1818 & 0.0228 \\
222 & 1.73 & 13.70 & 1.2 & 1828 & 0.0419 \\
223 & 1.73 & 11.80 & 1.4 & 1813 & 0.0710 \\
235 & 2.05 & 15.00 & 1.3 & 1878 & 0.0584 \\
231 & 2.29 & 15.00 & 1.5 & 1915 & 0.0786 \\
207 & 2.55 & 24.14 & 1.0 & 2009 & 0.0031 \\
225 & 2.55 & 22.00 & 1.1 & 1934 & 0.0237 \\
226 & 2.55 & 20.20 & 1.2 & 1883 & 0.0426 \\
208 & 2.55 & 17.43 & 1.4 & 1929 & 0.0715 \\
\hline
\end{tabular}

For validation of methane measurements were performed in a heated cell. The heated section of the absorption cell is $300 \mathrm{~mm}$ long with a $100 \mathrm{~mm}$ long quartz cell with wedged windows centered in the heated section. This ensures a homogeneous temperature profile along the absorption path. The temperature homogeneity within the cell is better than $2 \%$ measured with a thermocouple. The maximum temperature of the heated cell is $1173 \mathrm{~K}$. A total flow of $50 \mathrm{sccm}$ of methane/nitrogen mixtures were used to achieve different concentration levels of methane. The error in the concentration is estimated from the errors in the mass flow controllers to $5 \%$.

\section{RESULTS AND DISCUSSION}

\section{A. Sensor validation}

Validation measurements have been performed in laminar flat flames and a heated cell at atmospheric pressure. To compare the flame center temperatures at a distance of $15 \mathrm{~mm}$ above the burner surface with temperatures previously measured with CARS $[22,23]$ the measured line-of-sight spectra were Abelinverted using the method of Dasch[24] prior to fitting the absorption spectrum. Details of the procedure are provided in $[25,26]$. Temperatures agree very well within the measurement uncertainty of $2-3 \%$ for the absorption measurements and $2.5 \%$ for CARS (fig. 4a). Measured concentration levels of $\mathrm{CO}$ were compared with equilibrium values and were found to agree in general within the measurement accuracy (fig. 4b). Discrepancies for some flames could be attributed to differences in the flame conditions (i.e. errors in the mass flows, temperature and flow rate of the cooling water, ambient pressure).

For validation of methane laminar flat flames are not well suited because of the usually low concentration levels
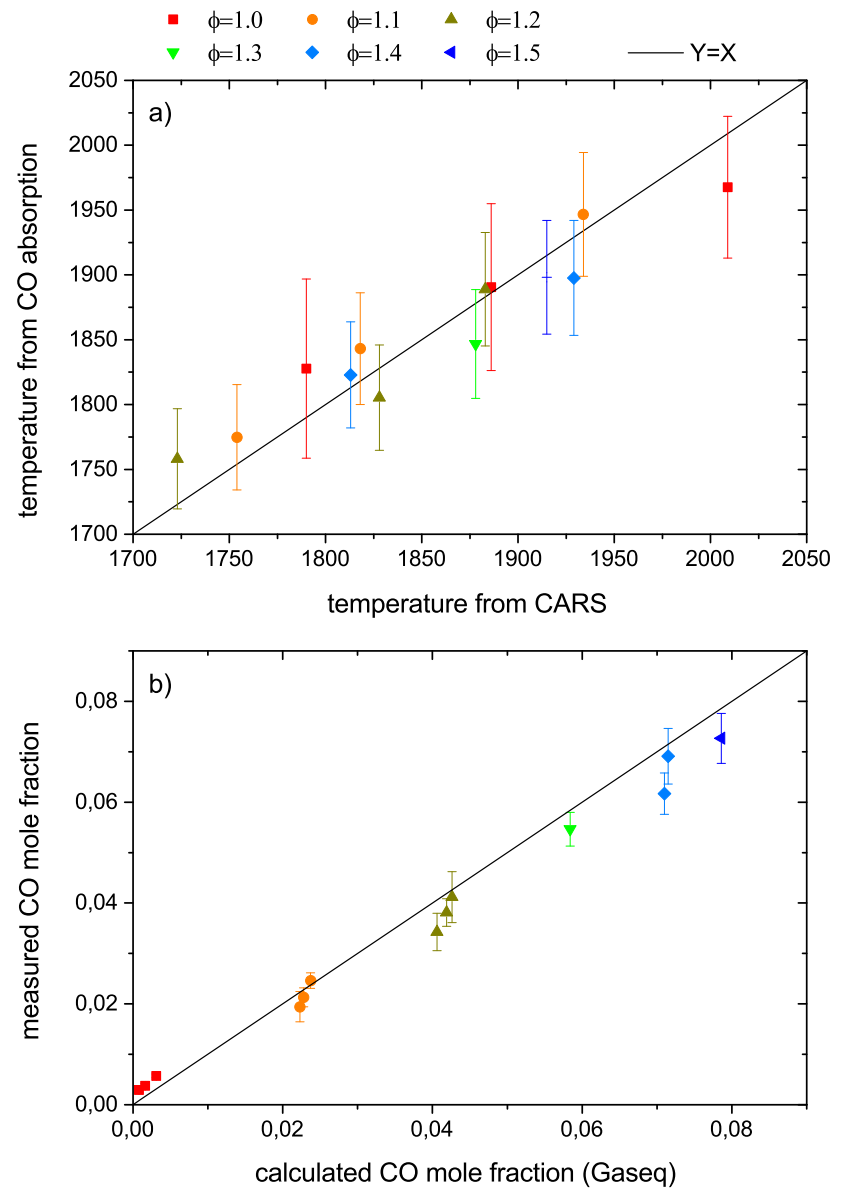

Fig. 4. Validation measurements for $\mathrm{CO}$ mole fraction (b) and temperature (a). Reference values for $\mathrm{T}$ and $\mathrm{CO}$ mole fraction are taken from [22].

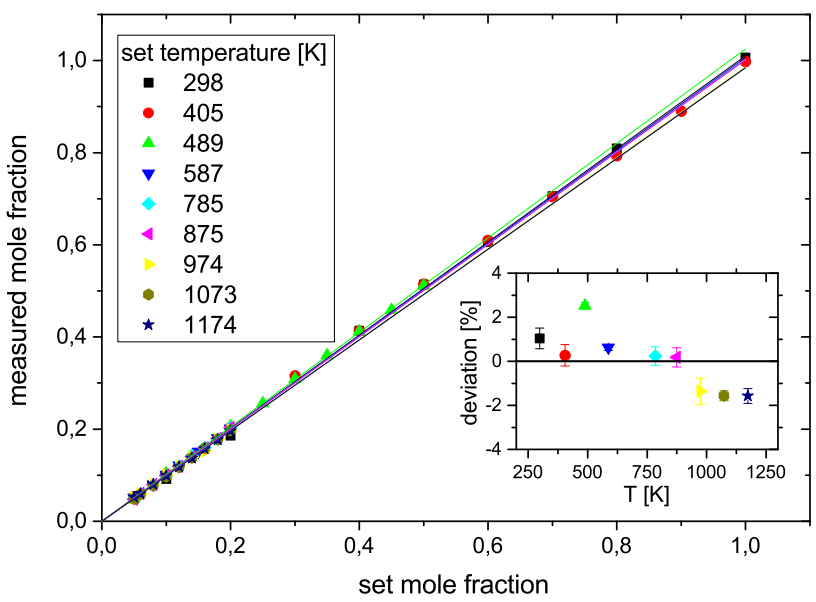

Fig. 5. Validation measurements of methane/nitrogen mixtures in a heated cell. The deviation in the inset is determined for each temperature from the deviation of the slope of the fitted line from the ideal slope of 1 . The plotted error bars are taken from the error in the fitted slopes. 
of methane. Therefore measurements were performed in a heated cell. Different concentration levels of methane in nitrogen were obtained by mixing methane and nitrogen with a total maximum gas flow of $50 \mathrm{sccm}$. For each temperature about 10 different concentration levels were used. At higher temperatures $(>500 \mathrm{~K})$ the maximum useable mole fraction was 0.2 to avoid too strong absorption. At each temperature a line was fitted to the measured mole fraction plotted against the set mole fraction (figure 5). The error in the fitted slopes is $<0.6 \%$ and the deviation from 1 is for all investigated temperatures below $3 \%$. This is an excellent agreement taking into account the uncertainty of the line strengths of $10-20 \%$.

\section{B. Gasifier measurements}

By moving the burner inside the reactor and by using two optical ports, measurements with distances from the burner inlet from $20 \mathrm{~mm}$ to $680 \mathrm{~mm}$ could be achieved. For the calculation of the absorption spectra with equation 1 a path length $l$ of $280 \mathrm{~mm}$ (the diameter of the reactor) was used. This results in line-of-sight averaged mole fractions and temperatures. In figure 6 absorption spectra are shown for $\mathrm{CO}$ and water at $2.3 \mu \mathrm{m}$ (a), water and methane at $3.1 \mu \mathrm{m} \mathrm{(b)} \mathrm{and}$ water and acetylene (c). The very good agreement between the simulated spectrum and measurement, and the high signal to noise ratio is clearly visible. The residual is dominated by inaccuracies in the line positions and the line shape model function. Acetylene could not be detected during the gasifier measurements. The simulated acetylene spectrum in figure $6 \mathrm{c}$ was calculated for a mole fraction of $0.1 \mathrm{vol} \%$ and the pressure broadening parameters from HITRAN for acetylene in air. This is the minimal detectable mole fraction at these conditions and mainly limited by the background absorption from water. It should be noted that the actual linewidth in the reactor is somewhat different from the calculated value, because the pressure broadening parameters are different from air. Though for estimation of the minimal detectable mole fraction this value should be sufficient. The $1 \sigma$ standard deviation of the residual in figure $6 c$ is 0.003 . In regions without absorption lines a $1 \sigma$ standard deviation of $4 \times 10^{-4}$ can be achieved (figure 6a). More precise knowledge of the shape of the water background could therefore increase the sensitivity and allow the detection of acetylene concentration levels of about $0.01 \mathrm{vol} \%$. To achieve this, the pressure broadening parameters of the main species in the gasifier must be determined (mainly $\mathrm{N}_{2}, \mathrm{H}_{2}, \mathrm{H}_{2} \mathrm{O}, \mathrm{CO}$ and $\mathrm{CO}_{2}$ ). Also the concentration of these molecules must be known to accurately calculate the line shapes. In this investigation we will therefore focus on the quantification of $\mathrm{CO}$, water and methane.

Gas temperatures were determined by fitting the $\mathrm{CO}$ absorption spectrum. For the given experiment it is valid to assume that water and methane are in thermal equilibrium with CO. Therefore the temperature determined from the $\mathrm{CO}$ spectrum was used as a fixed value for fitting the spectrum around $3.1 \mu \mathrm{m}$. Mole fraction and temperature profiles for condition 1 are shown in figure 7. Error bars include systematic errors, as explained in 2.A and measurement precision determined from the standard deviation of individual measurements (typically 500 laser ramps). For clarity only the error bars at $20 \mathrm{~mm}$ and $680 \mathrm{~mm}$ are shown. Due to higher fluctuations the standard deviation is higher at lower distances to the burner. Measured concentration profiles are relatively flat, due to the fact that the gasifier has a very large recirculation zone surrounding the flame in the center
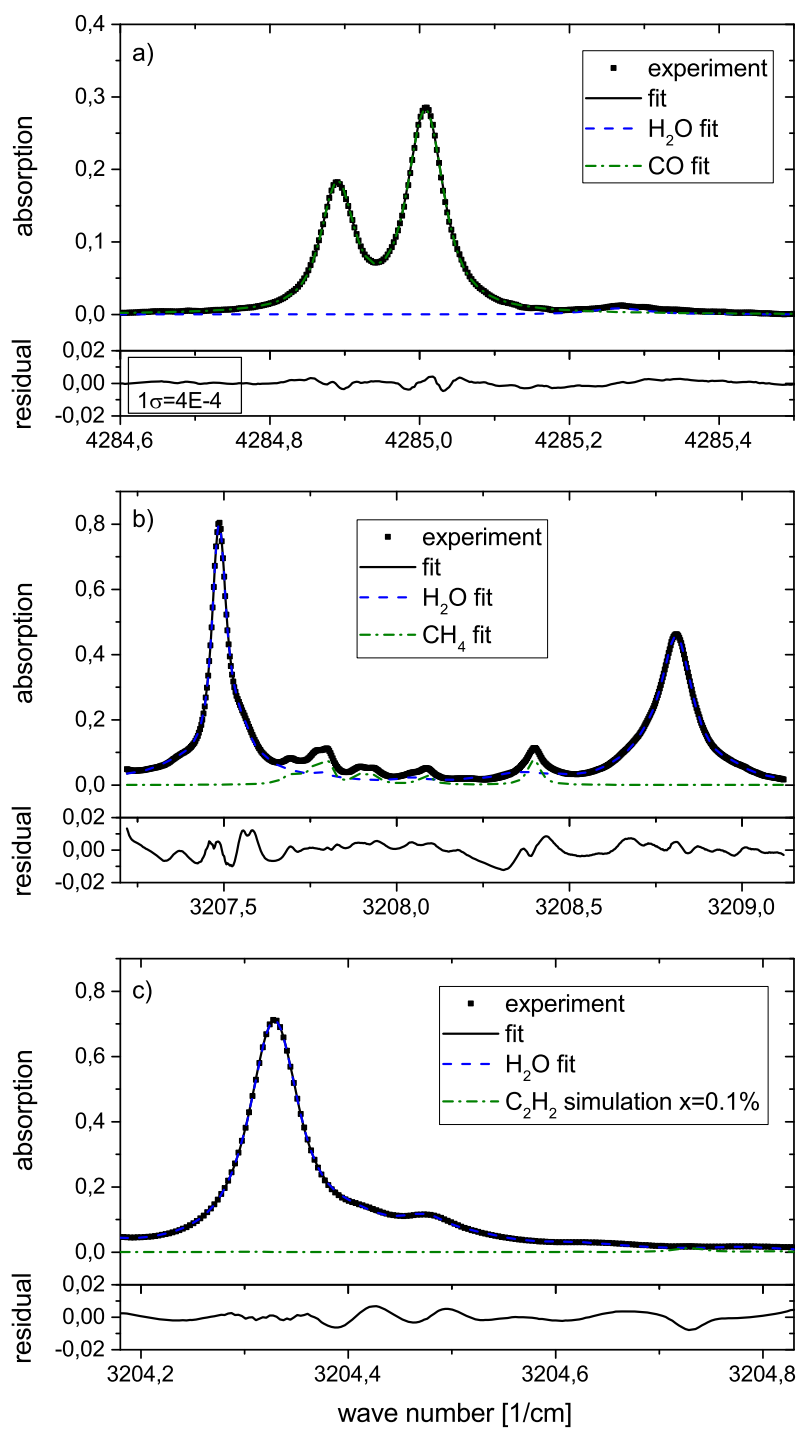

Fig. 6. Absorption spectra of $\mathrm{CO}$ and water (a), water and methane (b) and water and acetylene (c) measured in condition 2 . The acetylene spectrum (P31e line at $3204.7 \mathrm{~cm}^{-1}$ ) was simulated with pressure broadening parameters from HITRAN and a mole fraction of $0.1 \mathrm{vol} \%$, while for water, $\mathrm{CO}$ and methane the spectrum was fitted to the experiment. 
of the reactor. The absorption signal is therefore dominated by the recirculated reaction products. Methane concentration levels start to decrease after about $250 \mathrm{~mm}$ below the detection limit. The temperature profile shows a slow increase up to about $300 \mathrm{~mm}$. At higher distances the profile becomes also very flat and reaches a sort of plateau. In addition, a radial temperature profile has been measured with double bead type $\mathrm{B}$ thermocouples at $680 \mathrm{~mm}$. The average temperature of this measurement is $1647 \mathrm{~K}$ and is in good agreement with the temperature measured with absorption spectroscopy of $1634 \mathrm{~K}$. Details on the thermocouple measurement procedure can be found in [14].

\section{Comparison with model calculations}

Comparison of the line-of-sight integrated absorption measurements with model calculations is not as straight forward as for 2D imaging techniques like laser induced fluorescence or point measurements like LIBS. For condition 1 detailed large eddy simulations (LES) were performed which give 3D spatially resolved temperature and species information. Gas flows from the optical probes were neglected in the simulation. The reaction kinetics were computed by a detailed chemistry solver including 44 individual species and a direct calculation of 329 chemical reactions. The turbulence-chemistry interaction was accounted for by an assumed probability density function approach [27]. The unresolved sub-grid Reynolds stresses were calculated by the WALE (Wall-Adapting Local Eddy-viscosity) model [28]. The sub-processes pertaining to the liquid phase were computed by means of Lagrangian particle tracking. Dispersion and evaporation were modelled by variants of the dispersion model of Bini and Jones (2008) [29] and the evaporation model of Abramzon and Sirignano (1989) [30], respectively. The data were exchanged online between the gaseous Eulerian phase and the liquid Lagrangian phase via an iterative two-way-coupling procedure.

At a given distance from the burner these data can be averaged to compare these values with the values obtained with absorption spectroscopy. As long as the line strength of the lines is not very temperature sensitive and the temperature distribution along the absorption path is relatively uniform this should give reasonable good results. As can be seen in figure 7 the values for water and $\mathrm{CO}$ are slightly overestimated in the simulation but still within the measurement uncertainty of about $10-17 \%$ for water and $6-12 \%$ for CO. For methane the agreement is less good, but it should be noted that simulation of these low concentration levels is quite challenging. However temperatures are about $200 \mathrm{~K}$ too high in the simulation, which is well out of the measurement uncertainty of $2-3 \%$. Equilibrium calculations suggest an influence of about $50 \mathrm{~K}$ from the neglected nitrogen purge of the probes, which cannot explain the discrepancy. Therefore the difference might be due to neglected radiation and uncertainties in the chemical kinetics mechanism in the computation. Also the shape of the temperature profile is quite different. While the profile from the averaged simulation data shows a temperature peak at about $200 \mathrm{~mm}$, the measured profile does not show such a peak. In contrast it reaches a plateau after about $300 \mathrm{~mm}$.

Because the simple approach of averaging the LES data at a given distance to the burner gives unsatisfactory results a different approach has been used. Instead simple averaging of temperature and concentration values, absorption spectra have been simulated for every position along the absorption path

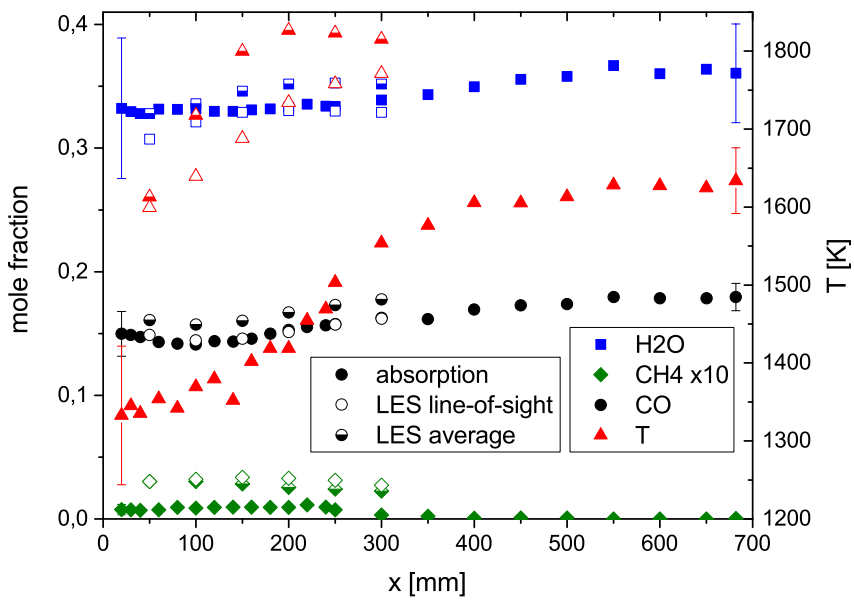

Fig. 7. Mole fraction and temperature profiles in the gasifier (condition 1). Closed symbols: results from the absorption measurements averaged over 500 individual laser scans. Error bars are only shown at $20 \mathrm{~mm}$ and $680 \mathrm{~mm}$ for clarity. Halfopen symbols: LES simulation averaged. Open symbols: LES simulation line-of-sight integrated.

based on the simulation data. The simulated spectra are then integrated along the line-of-sight, like during the absorption measurement. This way, inhomogeneities in temperature and mole fraction distribution are accounted for.

Another factor to take into consideration is the influence of the purge gas of the probes on the absorption measurements. The nitrogen exits the probe at the tip to keep the window clean from particles. Therefore the gas is diluted by the nitrogen near the probes. To estimate this influence measurements with LIBS have been performed using the same optical probe. The measured N/O-ratio was used to quantify the dilution. In figure 8 the measured oxygen volume fraction normalized by the value of the input mixture (compare table 1) is plotted against the distance from the reactor center. Close to the wall $(140 \mathrm{~mm})$ the dilution is about $40 \%$. The amount of dilution decays exponentially. At about $90 \mathrm{~mm}(50 \mathrm{~mm}$ from the reactor wall) almost no dilution can be measured. The influence on the effective absorption path length is about $6 \%$. However using an effective path length instead of the diameter of the reactor for data evaluation will only give correct results in case of a homogeneous species distribution. For example molecules only present in the reactor center will not be affected by the purge nitrogen at all. Because the species and temperature distributions along the line-of-sight cannot be measured with absorption spectroscopy the dilution factor, shown in figure 8 , was applied to the simulated profiles. Absorption spectra have been simulated based on the corrected mole fractions and temperatures from the LES simulation and integrated along the line-of-sight. The resulting spectra were evaluated in the same way as the measured absorption spectra.

The resulting temperatures and mole fractions are shown in figure 7 for condition 1 . As expected, values for water and $\mathrm{CO}$ are slightly lower compared to the simple averaged approach. Most notably the shape of the temperature profile now fits very well to the measured temperature profile. This is because the hot center of the reactor, were no $\mathrm{CO}$ is present in the simulation, does not contribute to the temperature measured with $\mathrm{CO}$ absorption. Therefore for comparison of absorption 


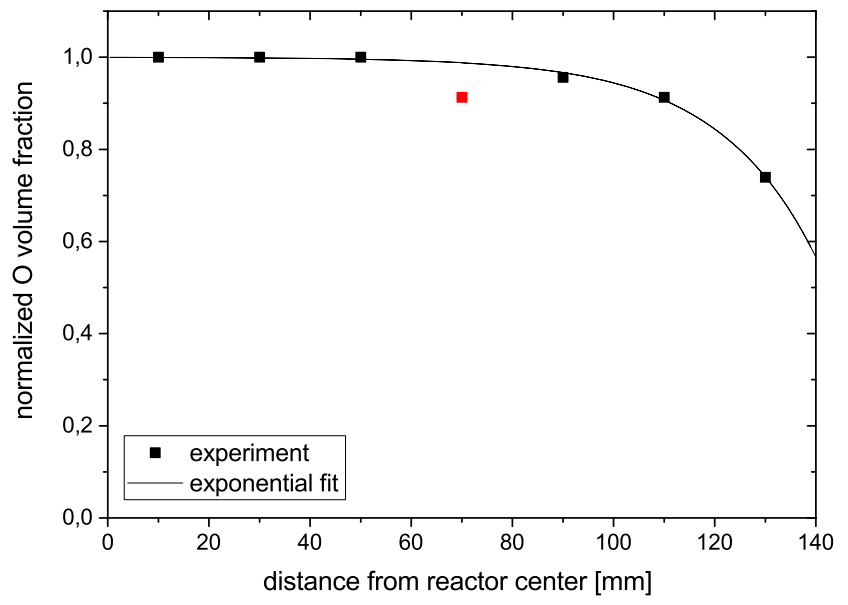

Fig. 8. Influence of the probe purge gas determined from the N/O ratio of LIBS measurements. The measured oxygen volume fraction was normalized to the value of the unreacted mixture and an exponential decay was fitted to the data. The data point at $70 \mathrm{~mm}$ was excluded from the fit.

and LES simulation the approach of considering the line-ofsight effect by modeling the absorption spectra gives better results. Although it should be noted that absolute temperatures are still $150-200 \mathrm{~K}$ too high. As mentioned above, this could be improved by taking into account cooling from the optical probe purge gas, including radiation in the computation and with improvements in the chemical kinetics mechanism.

\section{CONCLUSION}

A diode laser absorption spectrometer has been developed to measure species concentrations and temperatures in an atmospheric gasifier. Two wavelength regions have been selected: $2.3 \mu \mathrm{m}$ to measure $\mathrm{CO}$ and determine the temperature from the intensity ratio of the two lines and 3.1 $\mu \mathrm{m}$ to measure mainly methane and acetylene, but also water. At $2.3 \mu \mathrm{m}$ a tunable DFB diode laser was used and at $3.1 \mu \mathrm{m}$ a DFB interband cascade laser. Both laser beams were overlapped and coupled into one single mode fiber to guide the beam to the reactor. This way both wavelength regions can be measured simultaneously. Validation measurements have been performed in laminar flat flames and a heated cell. These measurements show good agreement between expected and measured concentrations and temperatures within the measurement accuracy.

Measurements in an atmospheric gasifier have been performed to obtain temperatures and concentrations at different distances to the burner. Acetylene concentrations were below the detection limit of about $0.1 \mathrm{vol} \%$, mainly limited by the background absorption of water. With more precise knowledge of the line positions and pressure broadening parameters the detection limit could be improved to $0.01 \mathrm{vol} \%$. Measured profiles were compared with current model calculations. For comparison of measurement and simulation the line-of-sight effect has been accounted for. This is necessary because temperature and species concentrations are not homogeneous along the absorption path. Also the influence from the optical probe on the measurement has been quantified and taken into account. While for $\mathrm{CO}$ and water a good agreement between measurement and simulation could be achieved, temperatures and methane mole fractions are overestimated in the simulation.

\section{FUNDING INFORMATION}

The authors gratefully acknowledge the financial support by the Helmholtz Association of German Research Centres (HGF) in the frame of the Helmholtz Virtual Institute for Gasification Technology - HVIGasTech (VH-VI-429).

\section{REFERENCES}

1. H. Teichert, T. Fernholz, and V. Ebert, "Simultaneous in situ measurement of $\mathrm{CO}, \mathrm{H} 2 \mathrm{O}$, and gas temperatures in a full-sized coalfired power plant by near-infrared diode lasers," Appl. Opt. 42, 20432051 (2003).

2. M. Lackner, G. Totschnig, G. Loeffler, H. Hofbauer, and F. Winter, "In-situ laser spectroscopy of $\mathrm{CO}, \mathrm{CH} 4$, and $\mathrm{H} 2 \mathrm{O}$ in a particle laden laboratory-scale fluidized bed combustor," Them. Sci 6, 13-27 (2002).

3. P. Ortwein, W. Woiwode, S. Fleck, M. Eberhard, T. Kolb, S. Wagner, M. Gisi, and V. Ebert, "Absolute diode laser-based in situ detection of $\mathrm{HCl}$ in gasification processes," Exp. Fluids 49, 961-968 (2010).

4. R. Sur, K. Sun, J. Jeffries, R. Hanson, R. Pummill, T. Waind, D. Wagner, and K. Whitty, "TDLAS-based sensors for in situ measurement of syngas composition in a pressurized, oxygen-blown, entrained flow coal gasifier," Appl. Phys. B 116, 33-42 (2014).

5. K. Sun, R. Sur, J. Jeffries, R. Hanson, T. Clark, J. Anthony, S. Machovec, and J. Northington, "Application of wavelength-scanned wavelength-modulation spectroscopy $\mathrm{H} 2 \mathrm{O}$ absorption measurements in an engineering-scale high-pressure coal gasifier," Appl. Phys. B 117, 411-421 (2014)

6. K. Sun, R. Sur, X. Chao, J. B. Jeffries, R. K. Hanson, R. J. Pummill, and K. J. Whitty, "TDL absorption sensors for gas temperature and concentrations in a high-pressure entrained-flow coal gasifier," Proc. Combust. Inst. 34, 3593 - 3601 (2013).

7. A. Sepman, Y. Ögren, M. Gullberg, and H. Wiinikka, "Development of TDLAS sensor for diagnostics of $\mathrm{CO}, \mathrm{H} 2 \mathrm{O}$ and soot concentrations in reactor core of pilot-scale gasifier," Appl. Phys. B 122, 1-12 (2016).

8. J. Simonsson, H. Bladh, M. Gullberg, E. Pettersson, A. Sepman, Y. Ögren, H. Wiinikka, and P.-E. Bengtsson, "Soot concentrations in an atmospheric entrained flow gasifier with variations in fuel and burner configuration studied using diode-laser extinction measurements," Energy Fuels 30, 2174-2186 (2016).

9. R. Sur, S. Wang, K. Sun, D. F. Davidson, J. B. Jeffries, and R. K. Hanson, "High-sensitivity interference-free diagnostic for measurement of methane in shock tubes," J. Quant. Spectrosc. Radiat. Transf. 156, $80-87$ (2015).

10. L. Hildebrandt and L. Nähle, "DFB laser diodes expand hydrocarbon sensing beyond $3 \mu \mathrm{m}$," Laser Focus World 48, 87-90 (2012).

11. L. Dong, F. K. Tittel, C. Li, N. P. Sanchez, H. Wu, C. Zheng, Y. Yu, A. Sampaolo, and R. J. Griffin, "Compact TDLAS based sensor design using interband cascade lasers for mid-IR trace gas sensing," Opt. Express 24, A528-A535 (2016).

12. L. S. Rothman, I. E. Gordon, R. J. Barber, H. Dothe, R. R. Gamache, A. Goldman, V. Perevalov, S. A. Tashkun, and J. Tennyson, "HITEMP, the high-temperature molecular spectroscopic database," J. Quant. Spectrosc. and Rad. Transfer 111, 2139-2150 (2010).

13. A. Sane, A. Satija, R. P. Lucht, and J. P. Gore, "Simultaneous CO concentration and temperature measurements using tunable diode laser absorption spectroscopy near $2.3 \mu \mathrm{m}$," Appl. Phys. B 117, 7-18 (2014).

14. S. Fleck, C. Hotz, P. Stoesser, and T. Kolb, "Gasification of biomassbased suspension fuels in an atmospheric entrained flow gasifier," in "Tagungsband 27. Deutscher Flammentag," (Clausthal-Zellerfeld, 2015).

15. S. Fleck, K. P. Geigle, C. Hotz, P. Kutne, and T. Kolb, "Formation and decay of hydrocarbon intermediates in an entrained flow gasifier," 
in "Tagungsband 27. Deutscher Flammentag," (Clausthal-Zellerfeld, 2015).

16. L. S. Rothman, I. E. Gordon, Y. Babikov, A. Barbe, D. Chris Benner, P. F. Bernath, M. Birk, L. Bizzocchi, V. Boudon, L. R. Brown, A. Campargue, K. Chance, E. A. Cohen, L. H. Coudert, V. M. Devi, B. J. Drouin, A. Fayt, J.-M. Flaud, R. R. Gamache, J. J. Harrison, J.-M. Hartmann, C. Hill, J. T. Hodges, D. Jacquemart, A. Jolly, J. Lamouroux, R. J. Le Roy, G. Li, D. A. Long, O. M. Lyulin, C. J. Mackie, S. T. Massie, S. Mikhailenko, H. S. P. Müller, O. V. Naumenko, A. V. Nikitin, J. Orphal, V. Perevalov, A. Perrin, E. R. Polovtseva, C. Richard, M. A. H. Smith, E. Starikova, K. Sung, S. Tashkun, J. Tennyson, G. C. Toon, V. Tyuterev, and G. Wagner, "The HITRAN2012 molecular spectroscopic database," J. Quant. Spectrosc. Radiat. Transf. 130, 4-50 (2013).

17. I. Stranic and R. K. Hanson, "Laser absorption diagnostic for measuring acetylene concentrations in shock tubes," J. Quant. Spectrosc. Radiat. Transf. 142, 58 - 65 (2014).

18. J. Humlicek, "Optimized computation of the voigt and complex probability functions," J. Quant. Spectrosc. Radiat. Transfer 27, 437444 (1982).

19. J. Gounder, P. Kutne, and W. Meier, "Development of a laser-induced plasma probe to measure gas phase plasma signals at high pressures and temperatures," Spectrochim. Acta, Part B 74-75, 66 - 73 (2012). 6th Euro-Mediterranean Symposium on Laser Induced Breakdown Spectroscopy (EMSLIBS 2011).

20. M. Ebenhoch, "Anwendung der laserinduzierten Plasmaspektroskopie (LIPS) zur Bestimmung des Mischungsbruchs in reaktiven Strömungen," Master's thesis, Institut für Verbrennungstechnik der Luft- und Raumfahrt (IVLR), Universität Stuttgart (2016).

21. C. Morley, "Gaseq," http://www.gaseq.co.uk/.

22. P. Weigand, R. Lückerath, and W. Meier, "Documentation of flat premixed laminar $\mathrm{CH} 4 /$ air standard flames: Temperatures and species concentrations," Institute of Combustion Technology, German Aerospace Center (DLR), http://www.dlr.de/vt/desktopdefault.aspx/tabid-3065/4632_read-6696/.

23. S. Prucker, W. Meier, and W. Stricker, "A flat flame burner as calibration source for combustion research: Temperatures and species concentrations of premixed $\mathrm{H}_{2}$ / air flames," Rev. Sci. Instrum. 65, 29082911 (1994).

24. C. J. Dasch, "One-dimensional tomography: a comparison of abel, onion-peeling, and filtered backprojection methods," Appl. Opt. 31, 1146-1152 (1992).

25. P. Nau, J. Koppmann, A. Lackner, K. Kohse-Höinghaus, and A. Brockhinke, "Quantum cascade laser-based MIR spectrometer for the determination of $\mathrm{CO}$ and $\mathrm{CO} 2$ concentrations and temperature in flames," Appl. Phys. B 118, 361-368 (2015).

26. R. Villarreal and P. Varghese, "Frequency-resolved absorption tomography with tunable diode lasers," Appl. Opt. 44, 6786-6795 (2005).

27. M. Di Domenico, "Numerical simulations of soot formation in turbulent flows," Ph.D. thesis, Fakultät für Luft- und Raumfahrttechnik und Geodäsie, Universität Stuttgart (2008).

28. F. Nicoud and F. Ducros, "Subgrid-scale stress modelling based on the square of the velocity gradient tensor," Flow Turbul. Combust. 62 , 183-200 (1999).

29. M. Bini and W. P. Jones, "Large-eddy simulation of particle-laden turbulent flows," J. Fluid Mech. 614, 207-252 (2008).

30. B. Abramzon and W. Sirignano, "Droplet vaporization model for spray combustion calculations," Int. J. Heat Mass Transfer 32, 1605-1618 (1989). 